\title{
Novel compound heterozygous mutations in the CHST6 gene cause macular corneal dystrophy in a Han Chinese family
}

\author{
Yanxia Huang ${ }^{1 \#}$, Lamei Yuan ${ }^{1 \#}$, Yanna $\mathrm{Cao}^{2}$, Renhong Tang ${ }^{2}$, Hongbo Xu ${ }^{1}$, Ziqian Tang ${ }^{1}$, Hao Deng $^{1,3,4}$ \\ ${ }^{1}$ Center for Experimental Medicine, the Third Xiangya Hospital, Central South University, Changsha, China; ${ }^{2}$ Department of Ophthalmology, \\ the Third Xiangya Hospital, Central South University, Changsha, China; ${ }^{3}$ Department of Neurology, the Third Xiangya Hospital, Central South \\ University, Changsha, China; ${ }^{4}$ Disease Genome Research Center, Central South University, Changsha, China \\ Contributions: (I) Conception and design: Y Huang, L Yuan, H Deng; (II) Administrative support: None; (III) Provision of study materials or \\ patients: Y Huang, L Yuan, Y Cao, R Tang, H Deng; (IV) Collection and assembly of data: Y Cao, R Tang, H Xu, Z Tang; (V) Data analysis and \\ interpretation: Y Huang, L Yuan, Y Cao, R Tang, H Xu, H Deng; (VI) Manuscript writing: All authors; (VII) Final approval of manuscript: All \\ authors. \\ \#These authors contributed equally to this work. \\ Correspondence to: Hao Deng, MD, PhD. Distinguished Professor of the Lotus Scholars Award Program of Hunan Province, Distinguished Professor \\ of Sheng Hua Scholars Award Program of Central South University, Professor and Director of Center for Experimental Medicine, Professor of \\ Neurology, the Third Xiangya Hospital, Central South University, Professor and Director of Disease Genome Research Center, Central South \\ University, 138 Tongzipo Road, Changsha 410013, China. Email: hdeng008@yahoo.com.
}

Background: Macular corneal dystrophy (MCD), a rare autosomal recessive disorder, is caused by pathogenic mutations in the carbohydrate sulfotransferase 6 gene (CHST6) and is characterized by bilateral progressive stromal clouding and vision loss. Corneal transplantation is often necessary. This study aimed to identify disease-causing mutations in a Han-Chinese MCD patient.

Methods: A 37-year-old female diagnosed with MCD was recruited. The clinical materials were observed and described, and peripheral blood sample was extracted. Whole exome sequencing (WES) and Sanger sequencing were used to reveal genetic defects. The pathogenicity of identified mutations was assessed using in silico analysis.

Results: The patient had typical features of MCD, including decreased vision, multiple irregular graywhite corneal opacities, and corneal thinning. A novel nonsense mutation c.544C>T (p.Gln182Ter) and a validated missense mutation c.631C>G (p.Arg211Gly) were identified in the CHST6 gene coding region, both classified as "pathogenic" following the American College of Medical Genetics and Genomics standards and guidelines.

Conclusions: This study reports a Han-Chinese MCD patient with a novel nonsense mutation c.544C>T (p.Gln182Ter) and a recurrent missense mutation c.631C>G (p.Arg211Gly), which expand the spectrum of genetic mutations. The results of this study extend genotype-phenotype correlations between the CHST6 gene mutations and MCD clinical findings, contributing to a more accurate diagnosis and the development of potential gene-targeted MCD therapies.

Keywords: Carbohydrate sulfotransferase 6 gene (CHST6); compound heterozygous mutations; Han Chinese family; macular corneal dystrophy (MCD)

Submitted Oct 29, 2020. Accepted for publication Feb 04, 2021.

doi: $10.21037 /$ atm-20-7178

View this article at: http://dx.doi.org/10.21037/atm-20-7178 


\section{Introduction}

Macular corneal dystrophy (MCD, MIM 217800), a rare autosomal recessive corneal disease, is characterized by bilateral progressive scattered stromal clouding, irregular gray-white corneal opacities, and central corneal thinning. The late stage of vision loss requires corneal transplants (1-3). The prevalence of MCD is estimated to be $9.7 / 1,000,000$, with different distribution frequencies worldwide. The prevalence is $1.2 / 1,000,000$ and 7.6/100,000 in America and Iceland, respectively $(4,5)$. The relatively common occurrence in India, Saudi Arabia and Iceland is associated with the high frequency of consanguineous marriage, without involvement of specific environmental or systemic factors $(4,5)$. The onset is usually in the first decade and presents with central superficial irregular whitish corneal opacities. These opacities progressively expand to the corneal periphery, and even to the entire stroma with corneal thinning, resulting in severe vision impairment in middle age $(2,6-8)$. Therefore, early diagnosis and treatment are necessary for MCD. The histopathological characteristics of MCD are typically glycosaminoglycan accumulation in different corneal layers, including the epithelium, Bowman's membrane, the stroma, Descemet's membrane, the endothelium, and keratocytes $(3,7,9)$. The different onset ages, disease severity, and non-coding region mutations support that the gene expression may be regulated by epigenetic factor(s) $(2,7,9)$.

In 2000 , coding mutations in the carbohydrate sulfotransferase 6 gene (CHST6, MIM 605294) and rearrangements in the upstream region of the CHST6 gene were reported as being responsible for MCD $(10,11)$. The gene encodes corneal glucosamine $\mathrm{N}$-acetyl-6sulfotransferase (C-GlcNAc6ST), which contains an $\mathrm{N}$-terminal with a short cytosolic tail, a transmembrane domain, and a large Golgi luminal C-terminal domain, belonging to the carbohydrate sulfotransferase family. The C-terminal domain has sulfate donor 3'-phosphoadenosine5 '-phosphosulfate (PAPS) binding sites, a catalytic module and an area that determines carbohydrate specificity in vivo (12-14). The C-GlcNAc6ST functions in the formation of sulfated keratan sulfate (KS), which is transported to the extracellular matrix where it forms some components of the corneal stroma (15). CHST6 mutants lead to accumulation of poorly sulfated or unsulfated KS, resulting in corneal fibril organization malformations and progressive opacification $(14,16)$. Up to now, at least 193 CHST6 gene mutations, including missense, nonsense, deletion, insertion/duplication, deletion-insertion, and complex rearrangement, have been reported in different patients with MCD $(1,14,17,18)$. The homozygous or compound heterozygous mutations in CHST6 gene might be the potential early MCD diagnosis markers (2,3,8-17).

We present the following article in accordance with the MDAR reporting checklist (available at http://dx.doi. org/10.21037/atm-20-7178).

\section{Methods}

\section{Subjects and clinical evaluation}

A 37-year-old female diagnosed with MCD was recruited from the Third Xiangya Hospital, Central South University (Changsha, China). The study was conducted in accord with the Declaration of the Helsinki (as revised in 2013) and approved by the Institutional Review Board of the Third Xiangya Hospital, Central South University, Changsha, China (No.: 2018-S400). Written informed consent was obtained from the female patient. Two ophthalmologists evaluated the patient who underwent a comprehensive ocular examination, including bestcorrected visual acuity (BCVA) tests, slit-lamp, confocal microscopy, and optical coherence tomography (OCT) examinations. The clinical diagnosis for MCD was based on the typical corneal features (1). The genomic DNA (gDNA) was isolated from peripheral blood sample of the patient using a phenol-chloroform extraction procedure, as previously described (19-21).

\section{Whole exome sequencing (WES)}

WES of the proband was performed by BGI Genomics, BGI-Shenzhen (Shenzhen, China), as previously described $(22,23)$. The quantified gDNA sample was randomly sheared into fragments by Covaris E220 (Covaris, Inc.) and achieved library fragments between $150 \mathrm{bp}$ and $250 \mathrm{bp}$. The DNA fragments were repaired by A-tailing reactions and then ligated with adapters. Ligation-mediated polymerase chain reaction (PCR) was used to amplify the fragments, and enrichment was performed after the purification and the hybridization to the exome array. The captured products were amplified by circularization. The qualified captured library was then loaded on the BGISEQ-500 platform. High-throughput sequencing was performed to ensure the average sequencing coverage. 


\section{Variants analysis}

Raw data from the sequencing platform were further filtered to obtain clean reads by a standard data-filtering process. The qualified clean reads were aligned to the human reference genome sequence by the BurrowsWheeler Aligner (BWA) software (v0.7.15). Picard tools (v2.5.0, https://broadinstitute.github.io/picard/) and Genome Analysis Toolkit (GATK, v3.7, https://www. broadinstitute.org/gatk/guide/best-practices) were used for marking and removing duplicate reads, Indel realignment, base recalibration and variant calling. The single nucleotide polymorphism effect (SnpEff, https://pcingola.github. io/SnpEff/) tool was applied for variant annotation and prediction, including 1000 Genomes Project, the Single Nucleotide Polymorphism Database (dbSNP) build 141, the National Heart, Lung, and Blood Institute-Exome Sequencing Project 6500 (NHLBI-ESP6500), and the 2,471 Chinese controls of the BGI in-house databases. The possible effects on protein function or structure of missense and nonsense variants were predicted by MutationTaster (http://www.mutationtaster.org/), Protein Variation Effect Analyzer (PROVEAN, http://provean.jcvi.org/index.php), and the Polymorphism Phenotyping v2 (PolyPhen-2, http:// genetics.bwh.harvard.edu/pph2/). The American College of Medical Genetics and Genomics (ACMG) guidelines for interpreting gene variants were used to classify the variants as "pathogenic", "likely pathogenic", "uncertain significance", "likely benign", and "benign" $(24,25)$.

\section{Sanger sequencing}

For the potential pathogenic variants identified by WES in the patient, Sanger sequencing was performed subsequently. Primers were designed for candidate variants with Primer3 (http://frodo.wi.mit.edu/primer3/) according to the sequences of the human reference genome (http:// genome.ucsc.edu/). The primers were then synthesized by Sangon Biotech (Shanghai) Co., Ltd. Primer sequences were designed as: 5'-GTGTGCAAGCCACTGTGC-3' and 5'-GTGGCTACGGCACACCTC-3'. The sequencing results were analyzed by using Chromas software (v2.01, Technelysium Pty Ltd., South Brisbane, Australia).

\section{Conservative analysis and structure modeling}

Basic Local Alignment Search Tool of National Center for Biotechnology Information (NCBI BLAST, http://
blast.st-va.ncbi.nlm.nih.gov/Blast.cgi) executed multiple sequence alignments for ten different species. The online CPHmodels-3.2 (http://www.cbs.dtu.dk/services/ CPHmodels/) and RaptorX tool (http://raptorx.uchicago. edu) predicted wild-type and mutant protein structures. PyMOL software (v2.3, Schrödinger, LLC, Portland, USA) was used to show the three-dimensional structures (19).

\section{Statistical analysis}

Pie charts were used for categorical data to show the percentage of various mutations in each group or the percentage of patients with CHST6 nonsense mutations in different continents and countries.

\section{Results}

\section{Clinical data}

The patient complained of bilateral blurred vision, discomfort, tearing, and photophobia as being present for more than twenty years. The patient's visual acuity was 0.06 in the right eye and 0.04 in the left eye. Vision was not significantly improved with prescription lens glasses. A slitlamp examination detected multiple, irregular, gray-white opacities from the center of the cornea to the peripheral limbus in the patient (Figure 1A,B). Confocal microscopy examinations revealed the hyper-reflectivity was present in the basal epithelium and the anterior stroma of cornea (Figure $1 C, D, E, F)$. Corneal images of OCT showed the subepithelial and anterior stromal deposits resulting in the thinning of the cornea (Figure $2 A, B, C, D$ ).

\section{WES and identification of pathogenic mutations}

Approximately 126.34 million clean reads and 116.27 million total effective reads were obtained, with $99.91 \%$ of the reads mapped to the human reference genome. The mean sequencing depth on target regions was 120.93 . The fraction of the target regions covered by $10 \times$ or more was $98.98 \%$. A total of 98,802 SNPs and 14,863 Indels were detected in the proband. The compound heterozygous variants (c.544C>T and c.631C>G) in the CHST6 gene, a disease-causing gene for MCD, were prosecuted as the disease-causing factors in the proband, which were absent in 2,471 controls. The c. $544 \mathrm{C}>\mathrm{T}$ variant resulted in a premature stop codon in codon 182 (p.Gln182Ter). The c.631C>G variant led to an arginine-to-glycine substitution (p.Arg211Gly). The 

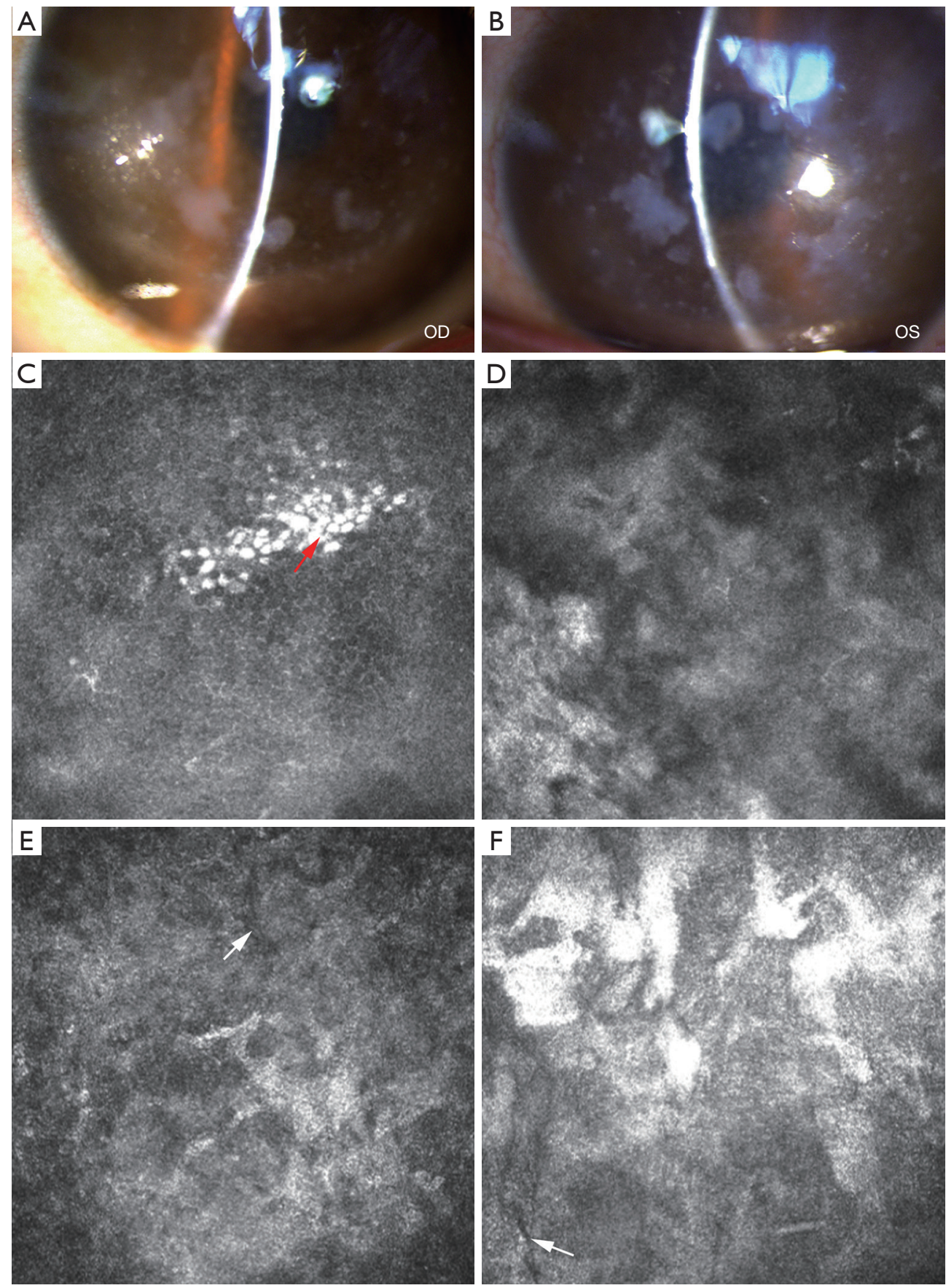

Figure 1 Slit-lamp and confocal microscopy photographs of the patient with macular corneal dystrophy. (A,B) Slit-lamp examination of the patient showed multiple irregular borders, gray-white opacities involving the center of the cornea, which extended to the limbus. (C) The confocal image at a depth of $39 \mu \mathrm{m}$. The basal epithelial layer appears to have numerous hyper-reflective cells (red arrow). (D) The confocal image at a depth of $50 \mu \mathrm{m}$. In the Bowman's layer, subbasal nerves with increased background were observed. (E) The confocal image at a depth of $83 \mu \mathrm{m}$. Hyper-reflective cells of the corneal stroma with multiple, small, black striae (white arrow). (F) The confocal image at a depth of $169 \mu \mathrm{m}$. The dark striae of the corneal stroma became large and wide along with the increased depth (white arrow). 

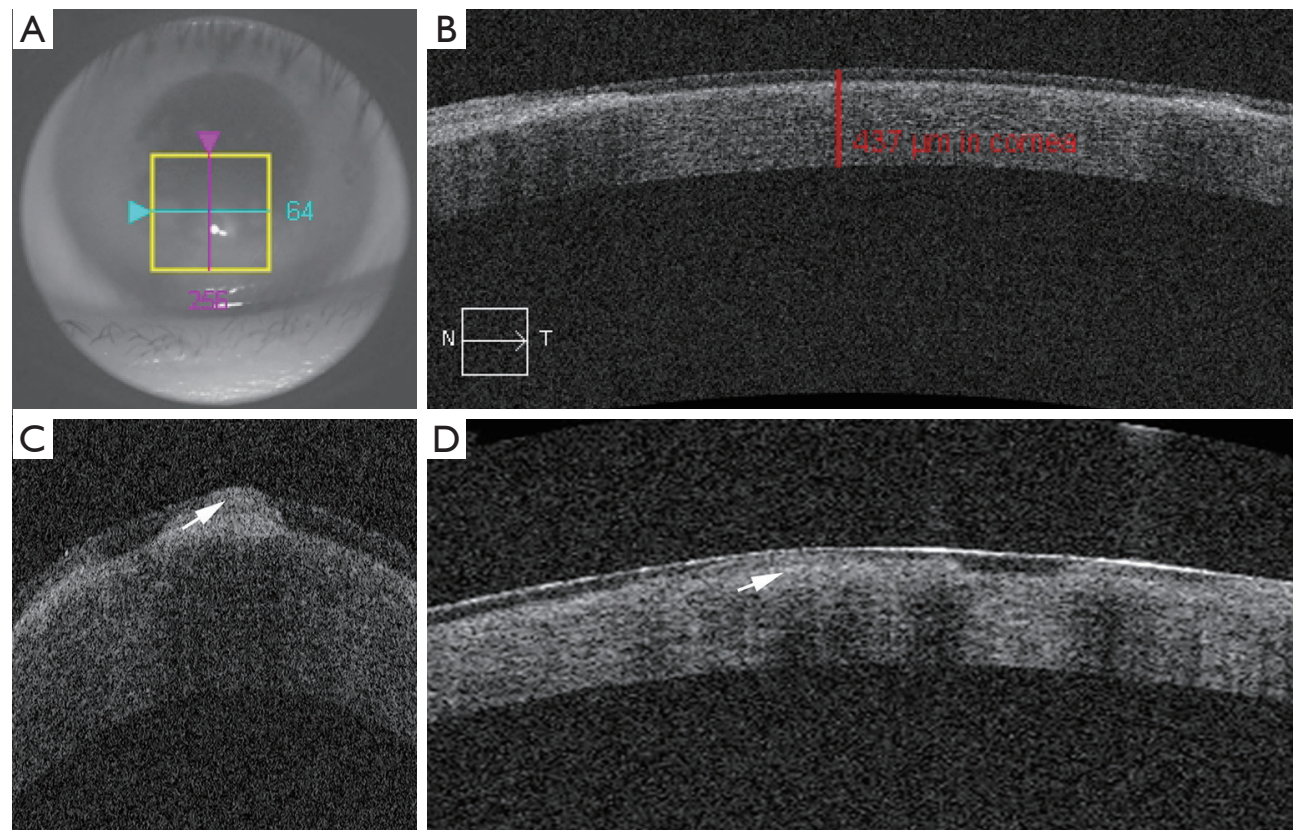

Figure 2 High-definition optical coherence tomography corneal imaging of the patient. (A) High-definition image of the right eye. (B) The central corneal thickness was $437 \mu \mathrm{m}$. (C) Large irregular subepithelial deposits (white arrow). (D) Diffuse deposits of hyper-reflectivity in the anterior stroma (white arrow).

prediction analyses of MutationTaster showed that the two variants, c. $544 \mathrm{C}>\mathrm{T}$ and c. $631 \mathrm{C}>\mathrm{G}$, were disease causing with the score near or equal to 1 . The variant, c. $631 \mathrm{C}>\mathrm{G}$, was predicted to be deleterious by PROVEAN (score: -6.73), damaging by SIFT (score: 0.001), and probably damaging by PolyPhen-2 (score: 1.000, specificity: 1.00, sensitivity: 0.00 ). The variants were classified as "pathogenic" mutations using the ACMG standards and guidelines for variant interpretation. The CHST6 compound heterozygous variants in the proband were further confirmed by Sanger sequencing (Figure $3 A, B, C$ ). Amino acid sequence analysis of the sulfotransferases of different species detected that arginine at mutant site (p.Arg211) was highly conserved (Figure 3D). Structural modeling showed the conformational alteration (Figure 3E).

\section{Discussion}

MCD is a stromal corneal dystrophy first described by ophthalmologist Oskar Fehr in 1904 (26). It is characterized by minute irregular gray-white fleck-like opacities deposited in the cornea during the first decade of life. It develops into diffuse multiple nodular opacities and results in severe vision loss over time $(7,27)$. Early diagnosis and following ophthalmologic evaluation, especially in children, should be beneficial for the patients in treatment and management (28).

In this study, a Han-Chinese patient with MCD is described, which has a novel nonsense mutation c.544C $>\mathrm{T}$ (p.Gln182Ter) and a validated missense mutation c.631C $>\mathrm{G}$ (p.Arg211Gly) in the CHST6 gene detected by both WES and Sanger sequencing. Compound heterozygous mutations may induce the production of truncated and single amino acid altered proteins and result in a typical MCD phenotype. Corneal phenotypes evaluated by slitlamp, confocal microscopy, and OCT showed no obvious differences between the patient of this study and other previously reported MCD cases, regardless of the mutations involved (9).

The CHST6 gene, located at chromosome 16q23.1, contains 3 exons and encodes a 395 -amino acid protein with a molecular weight of $44 \mathrm{kDa}(1,13)$. To date, various CHST6 gene mutations in MCD patients have been reported in different ethnic populations (Figure 4), which suggests strong allelic heterogeneity. In this study, a novel CHST6 nonsense mutation (p.Gln182Ter) in the female patient was identified, which is located in the critical sulfotransferase domain and may generate a truncated and dysfunctional protein product. Nonsense mutations 

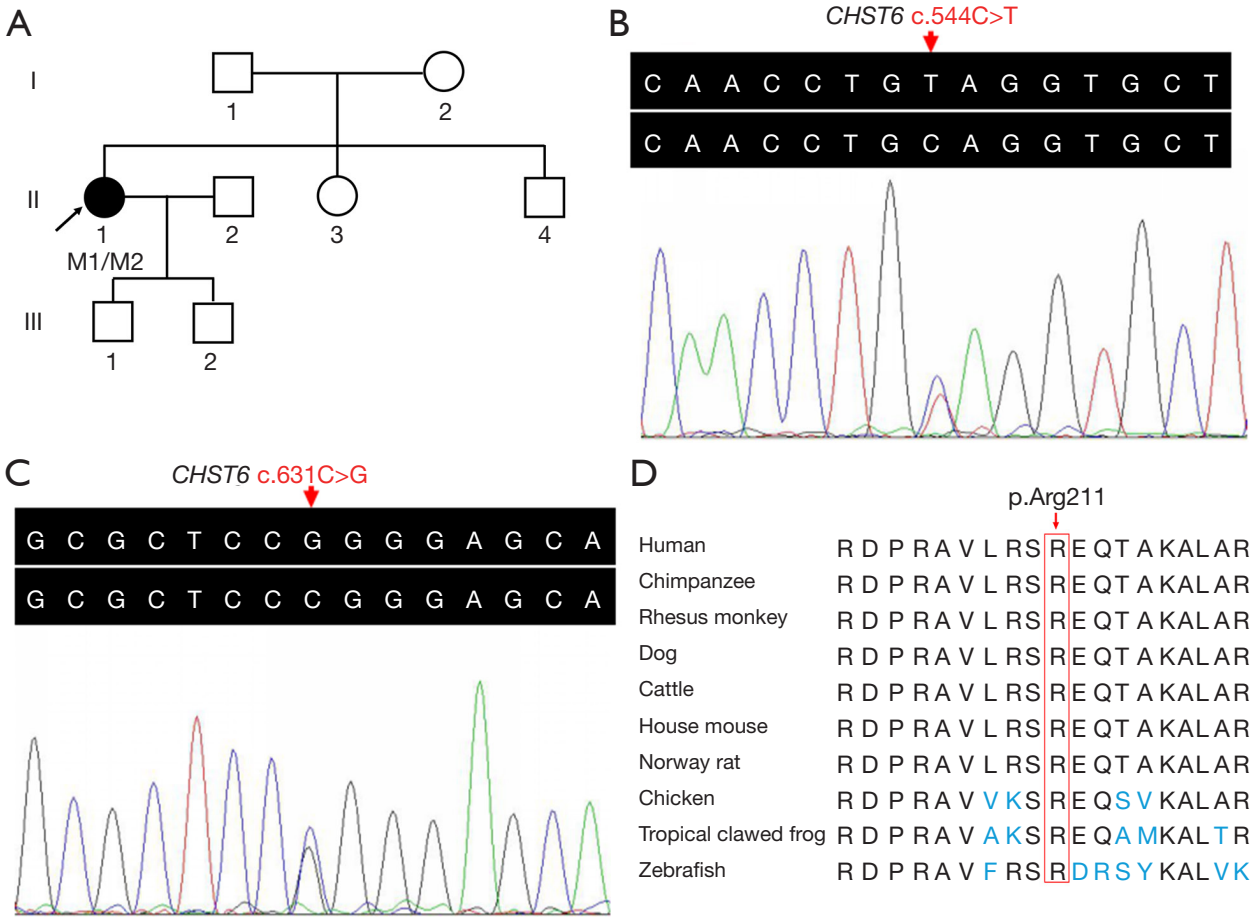

D
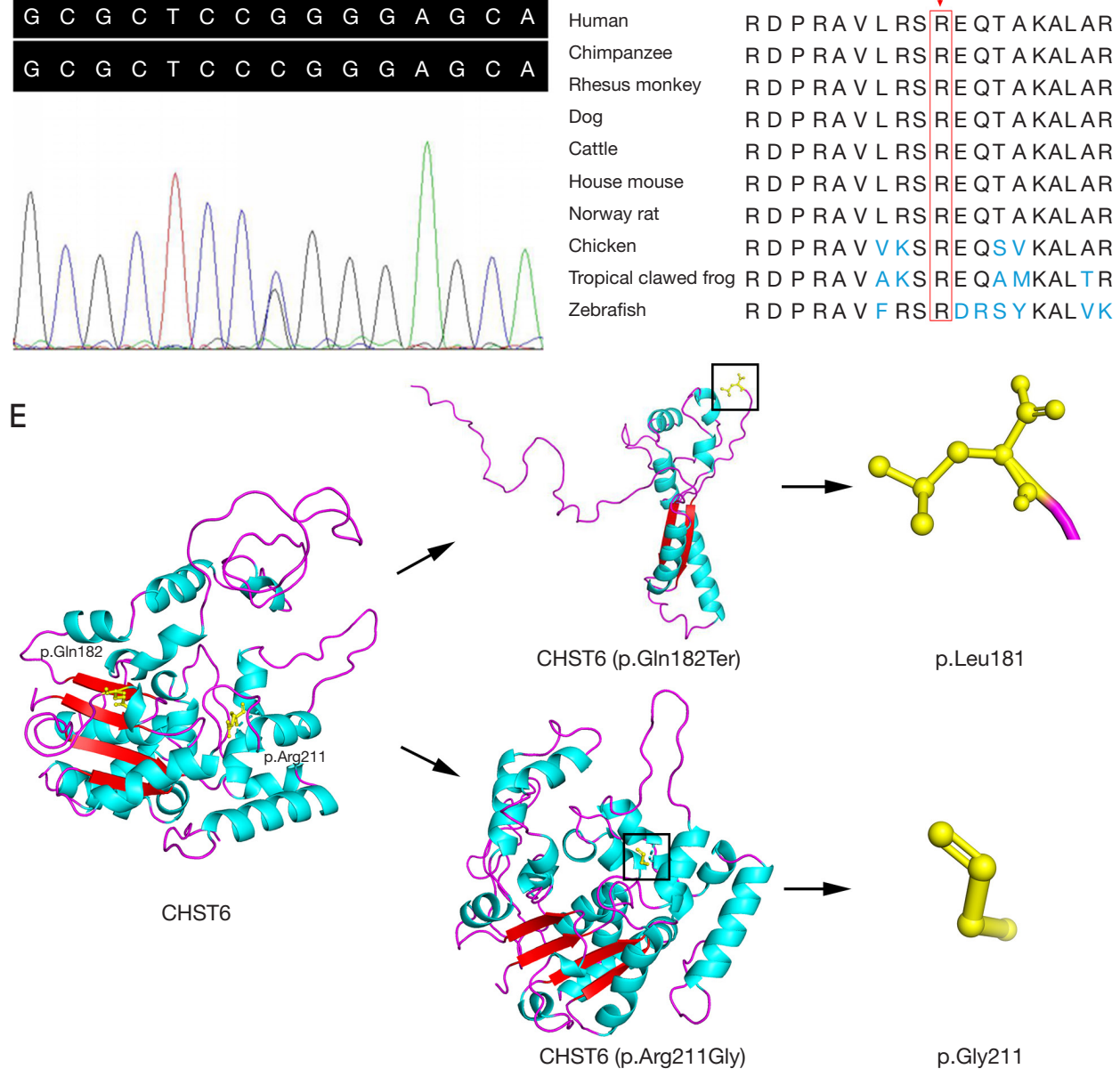

Figure 3 The identified variants of the CHST6 gene in the MCD family. (A) Pedigree figure. The arrow indicates the proband. M1, the allele with the CHST6 c.544C>T mutation; M2, the allele with the CHST6 c.631C>G mutation. (B,C) Patient (II:1) with the compound heterozygous mutations c.544C>T and c.631C>G in the CHST6 gene. (D) Conservation analysis of the CHST6 p.Arg211 amino acid residue. (E) Cartoon models of the CHST6 proteins. The wild-type glutamine at position 182, arginine at position 211 , leucine at position 181 before the premature truncation, and mutated glycine at position 211 are shown in ball-and-stick models by the PyMOL based on the CPHmodels and RaptorX. CHST6, the carbohydrate sulfotransferase 6 gene; MCD, macular corneal dystrophy. 


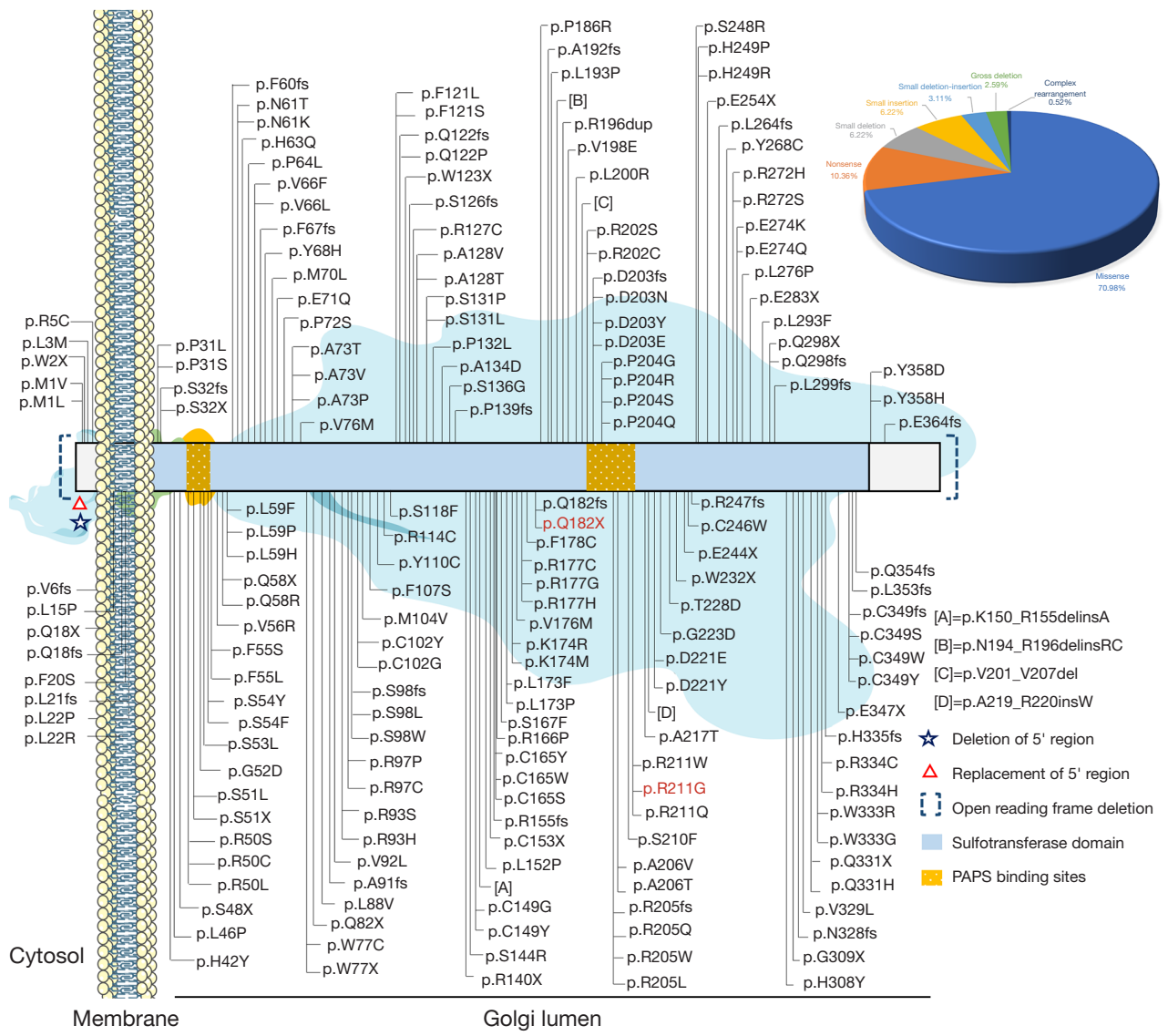

Figure 4 The mutations identified in the CHST6 gene associated with MCD. The schematic diagram annotated with the sulfotransferase domain (residue 42-356) and PAPS binding sites (residue 49-55 and 202-210) and model structure of the CHST6 protein were shown (UniProtKB: Q9GZX3). Small deletion, insertion and deletion-insertion are defined as those changes involving 20 bp or less according to the definition in Human Gene Mutation Database (http://www.hgmd.cf.ac.uk/ac/index.php). CHST6, the carbohydrate sulfotransferase 6 gene; MCD, macular corneal dystrophy; PAPS, 3'-phosphoadenosine-5'-phosphosulfate.

accounted for $10.36 \%$ of CHST6 alterations (Figure 4), in which Asian patients comprised a great proportion. For example, ethnic Chinese and Indian sufferers occupied $53.13 \%$ and $17.19 \%$, respectively (Table 1, Figure 5).

Genetic mutation type and position may influence CHST6 functions, particularly conserved positions across either or both of carbohydrate sulfotransferases or within a critical domain important for enzyme activity (40). The c.631C>G (p.Arg211Gly) mutation, previously reported as unique in two other Chinese families, was identified as one of the disease-causing alleles in our patient $(10,31)$. The identical nucleotide substitution observed in the other two families of the same ethnicity may indicate a founder effect $(10,31)$. The c. $631 \mathrm{C}>\mathrm{G}$ variant leads to the arginine-to-glycine substitution, changing from a positively charged basic amino acid residue to a neutral amino acid. The c.632G>A (p.Arg211Gln) variant has been identified in ethnic Vietnamese, Chinese, and German pedigrees $(3,10,44)$. The c.631C > T (p.Arg211Trp) variant has been found in ethnic Turkish and Japanese populations $(16,45)$. This multiple-ethnicity presence supports a conclusion that amino acid position 211 may be a hot spot mutation position (Table 2).

Cornea deposition was detected by confocal microscopy and was consistent in several MCD patients $(9,47)$. Highdefinition OCT showed some larger diffuse opacities and a hyper-reflective background located on the subepithelial. Multifocal hyper-reflective deposits were detected in the anterior stroma. The OCT results indicated that hyperreflective deposits caused thickening of the subepithelial 
Table 1 Nonsense mutations in the CHST6 gene associated with MCD

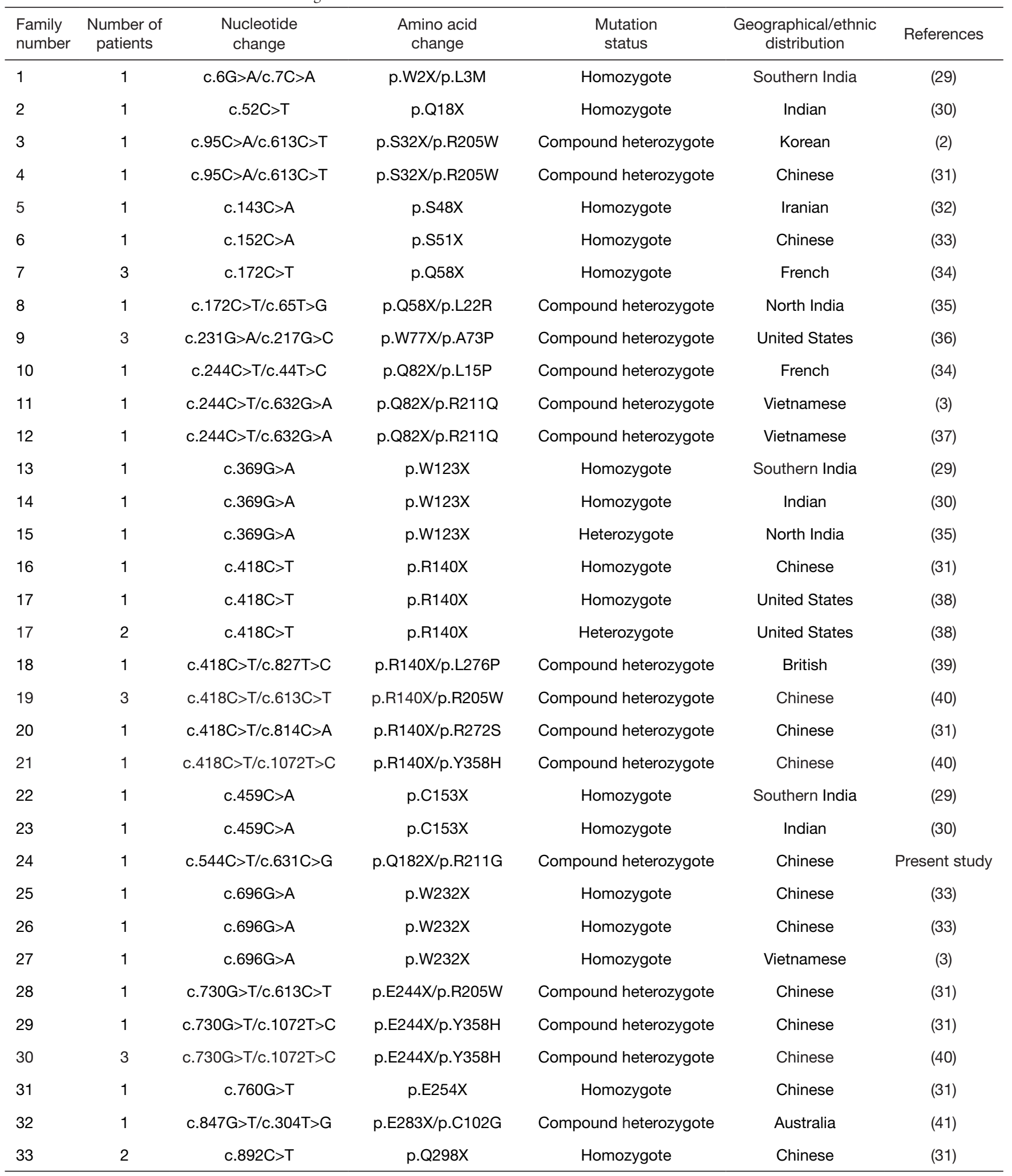

Table 1 (continued) 
Table 1 (continued)

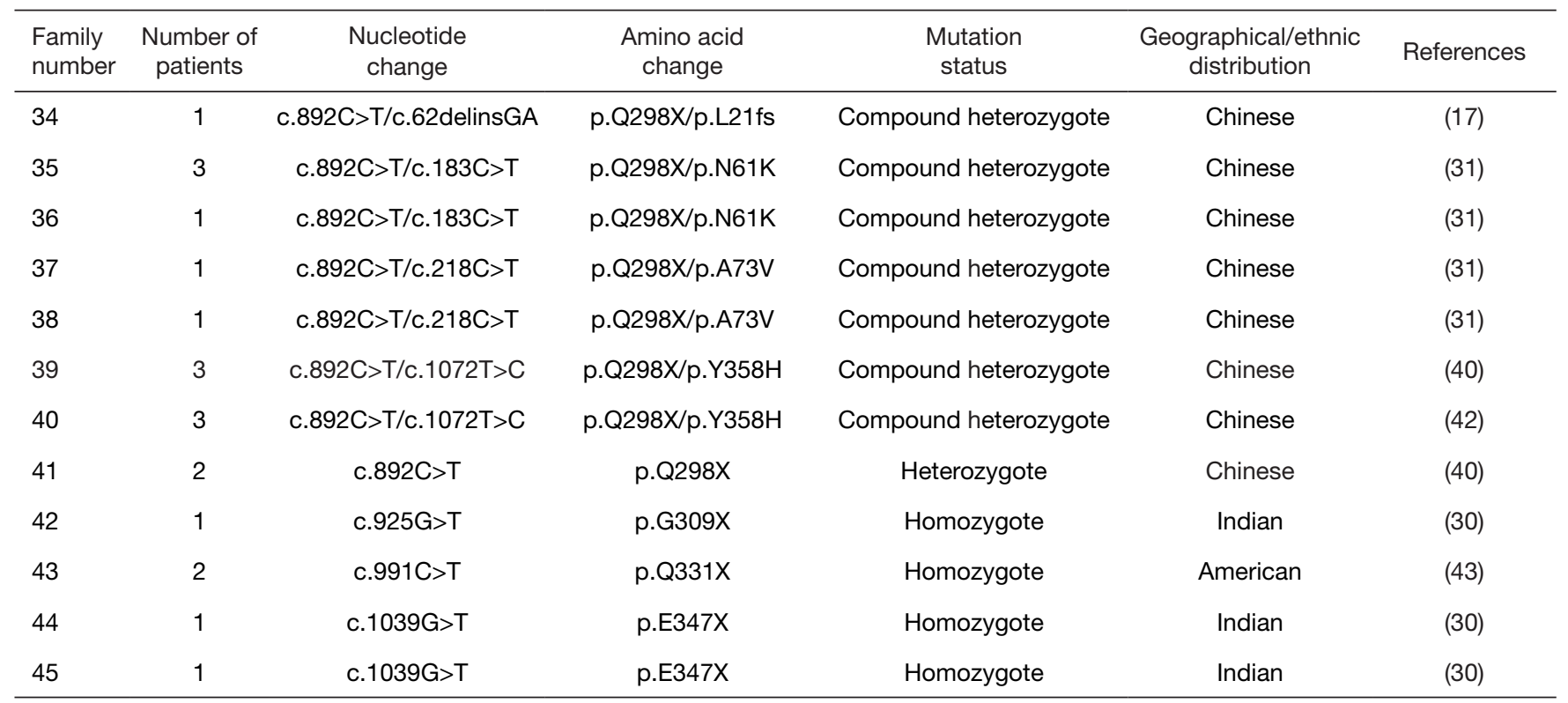

CHST6, the carbohydrate sulfotransferase 6 gene; MCD, macular corneal dystrophy.

A

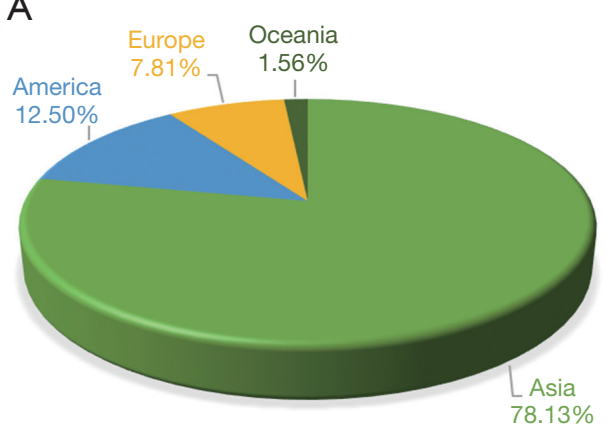

B

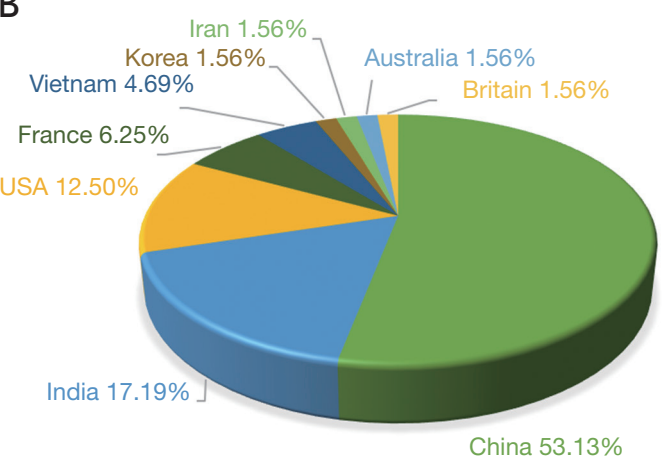

Figure 5 Distribution of CHST6 nonsense mutations with MCD. A pie chart showing the percentage of MCD patients carrying the CHST6 nonsense mutations in different continents (A) and countries (B). CHST6, the carbohydrate sulfotransferase 6 gene; MCD, macular corneal dystrophy.

and anterior stroma, which led to thinning of the cornea. Though MCD has been widely studied and various CHST6 mutations have been reported, the limited phenotypegenotype relationship data seem to have little value $(7,9)$. It has been reported that there may be more severe clinical phenotypes and earlier requirements of keratoplasty in ethnic German and French patients with CHST6 nonsense and frameshift mutations $(2,34,44)$. However, a few studies from Turkey, Egypt, and India have shown there are no consistent phenotypic differences between patients with missense mutations and truncating mutations $(12,16,30)$. More large-scale evaluations of MCD genotype-phenotype associations are warranted.

Different modalities of treatment are suggested for visual restoration of MCD (4,41). Phototherapeutic keratectomy treats superficial and anterior corneal pathology $(4,48)$. Penetrating keratoplasty may improve the BCVA of patients having corneal dystrophies without recurrence (44). Femtosecond laser-assisted lamellar keratectomy is reportedly successful in removing corneal opacities secondary to anterior 
Table 2 Missense mutation in codon 211 of the CHST6 gene in MCD patients

\begin{tabular}{|c|c|c|c|c|c|c|}
\hline $\begin{array}{l}\text { Family } \\
\text { number }\end{array}$ & $\begin{array}{l}\text { Number of } \\
\text { patients }\end{array}$ & Nucleotide change & Amino acid change & Mutation status & $\begin{array}{l}\text { Geographical/ethnic } \\
\text { distribution }\end{array}$ & References \\
\hline 1 & 1 & c. $631 \mathrm{C}>\mathrm{T}$ & p.R211W & Homozygote & NA & (11) \\
\hline 3 & 1 & c. $631 \mathrm{C}>\mathrm{T}$ & p.R211W & Homozygote & Japanese & $(45)$ \\
\hline 6 & 2 & c. $631 \mathrm{C}>\mathrm{T} / \mathrm{c} .649 \mathrm{G}>\mathrm{A}$ & p.R211W/p.A217T & Compound heterozygote & Japanese & $(45)$ \\
\hline 7 & 2 & c. $631 \mathrm{C}>\mathrm{G} / \mathrm{c} .445 \mathrm{~T}>\mathrm{G}$ & p.R211G/p.C149G & Compound heterozygote & Chinese & (31) \\
\hline 8 & 1 & c. $631 \mathrm{C}>\mathrm{G} / \mathrm{c} .544 \mathrm{C}>\mathrm{T}$ & p.R211G/p.Q182X & Compound heterozygote & Han Chinese & Present study \\
\hline 12 & 8 & c. $632 \mathrm{G}>\mathrm{A}$ & p.R211Q & Homozygote & Vietnamese & (3) \\
\hline 13 & 1 & c. $632 \mathrm{G}>\mathrm{A}$ & p.R211Q & Homozygote & Chinese & $(10)$ \\
\hline 14 & 1 & c. $632 \mathrm{G}>\mathrm{A} / \mathrm{c} .244 \mathrm{C}>\mathrm{T}$ & p.R211Q/p.Q82X & Compound heterozygote & Vietnamese & (3) \\
\hline 15 & 1 & c. $632 \mathrm{G}>\mathrm{A} / \mathrm{c} .244 \mathrm{C}>\mathrm{T}$ & p.R211Q/p.Q82X & Compound heterozygote & Vietnamese & $(37)$ \\
\hline 16 & 1 & c.632G>A/c.1045_1046del & p.R211Q/p.C349fs & Compound heterozygote & German & $(44)$ \\
\hline 17 & 1 & c. $632 \mathrm{G}>\mathrm{A} / \mathrm{c} .529 \mathrm{C}>\mathrm{G}$ & p.R211Q/p.R177G & Compound heterozygote & Vietnamese & $(46)$ \\
\hline
\end{tabular}

NA, not available; CHST6, the carbohydrate sulfotransferase 6 gene; MCD, macular corneal dystrophy.

corneal dystrophies (48). Newer therapy strategies such as gene transfer and gene editing have been evidenced to be effective in both in vitro and experimental models $(4,14)$.

In summary, a novel nonsense mutation p.Gln182Ter and a recurrent missense mutation p.Arg211Gly in the CHST6 gene were identified in a Han-Chinese patient with MCD. This finding expands the spectrum of genetic mutations. The discovery of additional CHST6 mutations in diverse ethnic populations will help to further explicate genotypephenotype associations. Additional functional investigations of specific mutations both in vitro and/or in vivo, such as in human corneas or constructed genetic-deficient animal models, will contribute to elucidate the underlying mechanisms in MCD.

\section{Acknowledgments}

We would like to thank the participating members and investigators for their cooperation and efforts in collecting clinical data, genetic information, and DNA specimens.
Funding: This work was funded by National Natural Science Foundation of China (81873686, 81670216 and 81800219), Natural Science Foundation of Hunan Province (2019JJ50927, 2020JJ3057, and 2020JJ4830), College Students' Innovative Training Plan Program (XCX20190529), Special Emergency Project from Science and Technology Department of Hunan Province (2020SK3032), and Distinguished Professor of the Lotus Scholars Award Program of Hunan Province, China.

\section{Footnote}

Reporting Checklist: The authors have completed the MDAR reporting checklist. Available at http://dx.doi.org/10.21037/ atm-20-7178

Data Sharing Statement: Available at http://dx.doi. org/10.21037/atm-20-7178

Conflicts of Interest: All authors have completed the ICMJE uniform disclosure form (available at http://dx.doi. 
org/10.21037/atm-20-7178). The authors have no conflicts of interest to declare.

Ethical Statement: The authors are accountable for all aspects of the work in ensuring that questions related to the accuracy or integrity of any part of the work are appropriately investigated and resolved. The study was conducted in accordance with the Declaration of Helsinki (as revised in 2013). The study was approved by the Institutional Review Board of the Third Xiangya Hospital, Central South University, China (No.: 2018-S400). Written informed consent was obtained from the female patient.

Open Access Statement: This is an Open Access article distributed in accordance with the Creative Commons Attribution-NonCommercial-NoDerivs 4.0 International License (CC BY-NC-ND 4.0), which permits the noncommercial replication and distribution of the article with the strict proviso that no changes or edits are made and the original work is properly cited (including links to both the formal publication through the relevant DOI and the license). See: https://creativecommons.org/licenses/by-nc-nd/4.0/.

\section{References}

1. Zhang J, Wu D, Li Y, et al. A comprehensive evaluation of 181 reported CHST6 variants in patients with macular corneal dystrophy. Aging (Albany NY) 2019;11:1019-29.

2. Park SH, Ahn YJ, Chae H, et al. Molecular analysis of the CHST6 gene in Korean patients with macular corneal dystrophy: identification of three novel mutations. Mol Vis 2015;21:1201-9.

3. Ha NT, Chau HM, Cung le X, et al. Mutation analysis of the carbohydrate sulfotransferase gene in Vietnamese with macular corneal dystrophy. Invest Ophthalmol Vis Sci 2003;44:3310-6.

4. Singh S, Das S, Kannabiran C, et al. Macular corneal dystrophy: an updated review. Curr Eye Res 2020:1-6.

5. Aggarwal S, Peck T, Golen J, et al. Macular corneal dystrophy: a review. Surv Ophthalmol 2018;63:609-17.

6. Bron AJ. Genetics of the corneal dystrophies: what we have learned in the past twenty-five years. Cornea 2000;19:699-711.

7. Rubinstein Y, Weiner C, Einan-Lifshitz A, et al. Macular corneal dystrophy and posterior corneal abnormalities.
Cornea 2016;35:1605-10.

8. Carstens N, Williams S, Goolam S, et al. Novel mutation in the CHST6 gene causes macular corneal dystrophy in a black South African family. BMC Med Genet 2016;17:47.

9. Nowinska AK, Wylegala E, Teper S, et al. Phenotype and genotype analysis in patients with macular corneal dystrophy. Br J Ophthalmol 2014;98:1514-21.

10. Zhang J, Wu D, Li Y, et al. Novel mutations associated with various types of corneal dystrophies in a Han Chinese population. Front Genet 2019;10:881.

11. Akama TO, Nishida K, Nakayama J, et al. Macular corneal dystrophy type I and type II are caused by distinct mutations in a new sulphotransferase gene. Nat Genet 2000;26:237-41.

12. El-Ashry MF, Abd El-Aziz MM, Shalaby O, et al. Molecular genetic study of Egyptian patients with macular corneal dystrophy. Br J Ophthalmol 2010;94:250-5.

13. Musselmann K, Hassell JR. Focus on molecules: CHST6 (carbohydrate sulfotransferase 6; corneal N-acetylglucosamine-6-sulfotransferase). Exp Eye Res 2006;83:707-8.

14. Safari I, Baradaran-Rafii A, Issazadeh-Navikas S, et al. CHST6 mutations identified in Iranian MCD patients and CHST6 mutations reported worldwide identify targets for gene editing approaches including the CRISPR/Cas system. Int Ophthalmol 2020;40:2223-35.

15. Zheng T, Zhao C, Zhao B, et al. Impairment of the autophagy-lysosomal pathway and activation of pyroptosis in macular corneal dystrophy. Cell Death Discov 2020;6:85.

16. Yaylacioglu Tuncay F, Kayman Kurekci G, Guntekin Ergun S, et al. Genetic analysis of CHST6 and TGFBI in Turkish patients with corneal dystrophies: five novel variations in CHST6. Mol Vis 2016;22:1267-79.

17. Jing Y, Zhou Y, Wang C, et al. Establishment of a nonintegrate iPS cell line CSUASOi002-A, from urine-derived cells of a female patient with macular corneal dystrophy carrying compound heterozygous CHST6 mutations. Stem Cell Res 2019;41:101598.

18. Klintworth GK. Corneal dystrophies. Orphanet J Rare Dis 2009;4:7.

19. Yuan L, Guo Y, Yi J, et al. Identification of a novel GJA3 mutation in congenital nuclear cataract. Optom Vis Sci 2015;92:337-42.

20. Yuan L, Yi J, Lin Q, et al. Identification of a PRX variant in a Chinese family with congenital cataract by exome sequencing. QJM 2016;109:731-5. 
21. Yuan L, Deng X, Song $Z$, et al. Genetic analysis of the RAB39B gene in Chinese Han patients with Parkinson's disease. Neurobiol Aging 2015;36:2907.e11-2.

22. Wang M, Guo Y, Rong P, et al. COL1A2 p.Gly1066Val variant identified in a Han Chinese family with osteogenesis imperfecta type I. Mol Genet Genomic Med 2019;7:e619.

23. Xia H, Huang $\mathrm{X}, \mathrm{Xu} \mathrm{H}$, et al. GJB2 c.235delC variant associated with autosomal recessive nonsyndromic hearing loss and auditory neuropathy spectrum disorder. Genet Mol Biol 2019;42:48-51.

24. Xiang Q, Yuan L, Cao Y, et al. Identification of a heterozygous mutation in the TGFBI gene in a HuiChinese family with corneal dystrophy. J Ophthalmol 2019;2019:2824179.

25. Richards S, Aziz N, Bale S, et al. Standards and guidelines for the interpretation of sequence variants: a joint consensus recommendation of the American College of Medical Genetics and Genomics and the Association for Molecular Pathology. Genet Med 2015;17:405-24.

26. Fehr O. Ein Fall von gittriger Hornhauttrübung. Centralbl Augenheilkd 1904;28:173-6.

27. Weiss JS, Møller HU, Aldave AJ, et al. IC3D classification of corneal dystrophies--edition 2. Cornea 2015;34:117-59.

28. Rødahl E, Knappskog PM, Bredrup C, et al. Congenital stromal corneal dystrophy 2008. In: Adam MP, Ardinger HH, Pagon RA, et al. editors. GeneReviews ${ }^{\circledR}$ [Internet]. Seattle, WA: University of Washington, 1993-2021.

29. Sultana A, Sridhar MS, Klintworth GK, et al. Allelic heterogeneity of the carbohydrate sulfotransferase- 6 gene in patients with macular corneal dystrophy. Clin Genet 2005;68:454-60.

30. Sultana A, Sridhar MS, Jagannathan A, et al. Novel mutations of the carbohydrate sulfotransferase-6 (CHST6) gene causing macular corneal dystrophy in India. Mol Vis 2003;9:730-4.

31. Liu Z, Tian X, Iida N, et al. Mutation analysis of CHST6 gene in Chinese patients with macular corneal dystrophy. Cornea 2010;29:883-8.

32. Birgani SA, Salehi Z, Houshmand M, et al. Novel mutations of CHST6 in Iranian patients with macular corneal dystrophy. Mol Vis 2009;15:373-7.

33. Huo YN, Yao YF, Yu P. Pathogenic mutations of TGFBI and CHST6 genes in Chinese patients with Avellino, lattice, and macular corneal dystrophies. J Zhejiang Univ
Sci B 2011;12:687-93.

34. Niel F, Ellies P, Dighiero P, et al. Truncating mutations in the carbohydrate sulfotransferase 6 gene (CHST6) result in macular corneal dystrophy. Invest Ophthalmol Vis Sci 2003;44:2949-53.

35. Paliwal P, Sharma A, Tandon R, et al. Molecular genetic analysis of macular corneal dystrophy patients from North India. Ophthalmic Res 2012;48:28-32.

36. Klintworth GK, Smith CF, Bowling BL. CHST6 mutations in North American subjects with macular corneal dystrophy: a comprehensive molecular genetic review. Mol Vis 2006;12:159-76.

37. Ha NT, Chau HM, Cung le X, et al. Identification of novel mutations of the CHST6 gene in Vietnamese families affected with macular corneal dystrophy in two generations. Cornea 2003;22:508-11.

38. Liu NP, Bao W, Smith CF, et al. Different mutations in carbohydrate sulfotransferase 6 (CHST6) gene cause macular corneal dystrophy types I and II in a single sibship. Am J Ophthalmol 2005;139:1118-20.

39. El-Ashry MF, Abd El-Aziz MM, Shalaby O, et al. Novel CHST6 nonsense and missense mutations responsible for macular corneal dystrophy. Am J Ophthalmol 2005;139:192-3.

40. Wang L, Tang X, Lv X, et al. CHST6 mutation screening and endoplasmatic reticulum stress in macular corneal dystrophy. Oncotarget 2017;8:96301-12.

41. Shields M, Craig J, Souzeau E, et al. Bilateral phototherapeutic keratectomy for corneal macular dystrophy in an adolescent: case report and review of the literature. Ophthalmic Genet 2020;41:368-72.

42. Dang X, Zhu Q, Wang L, et al. Macular corneal dystrophy in a Chinese family related with novel mutations of CHST6. Mol Vis 2009;15:700-5.

43. Aldave AJ, Yellore VS, Thonar EJ, et al. Novel mutations in the carbohydrate sulfotransferase gene (CHST6) in American patients with macular corneal dystrophy. Am J Ophthalmol 2004;137:465-73.

44. Gruenauer-Kloevekorn C, Braeutigam S, Heinritz W, et al. Macular corneal dystrophy: mutational spectrum in German patients, novel mutations and therapeutic options. Graefes Arch Clin Exp Ophthalmol 2008;246:1441-7.

45. Iida-Hasegawa N, Furuhata A, Hayatsu H, et al. Mutations in the CHST6 gene in patients with macular corneal dystrophy: immunohistochemical evidence of heterogeneity. Invest Ophthalmol Vis Sci 2003;44:3272-7.

46. Patel DA, Harocopos GJ, Chang SH, et al. Novel CHST6 
gene mutations in 2 unrelated cases of macular corneal dystrophy. Cornea 2011;30:664-9.

47. Kobayashi A, Fujiki K, Fujimaki T, et al. In vivo laser confocal microscopic findings of corneal stromal dystrophies. Arch Ophthalmol 2007;125:1168-73.

Cite this article as: Huang Y, Yuan L, Cao Y, Tang R, Xu H, Tang Z, Deng H. Novel compound heterozygous mutations in the CHST6 gene cause macular corneal dystrophy in a Han Chinese family. Ann Transl Med 2021;9(8):622. doi: 10.21037/ atm-20-7178
48. Steger B, Romano V, Biddolph S, et al. Femtosecond laser-assisted lamellar keratectomy for corneal opacities secondary to anterior corneal dystrophies: an interventional case series. Cornea 2016;35:6-13. 